\title{
Properties of Eucalyptus umbra Wood for Timber Structures
}

\author{
Marta C. J. A. Nogueira ${ }^{1}$, Diego H. Almeida ${ }^{2}$, Juliano S. Vasconcelos ${ }^{3}$, Tiago H. Almeida ${ }^{4}$, \\ Victor A. Araújo ${ }^{5}$, André L. Christoforo ${ }^{2, *}$, Francisco A. Rocco Lahr ${ }^{6}$ \\ ${ }^{1}$ Department of Architecture and Urbanism, Cuiabá, Brazil \\ ${ }^{2}$ Federal University of São Carlos, Department of Civil Engineering, São Carlos, Brazil \\ ${ }^{3}$ Research Group on Lignocellulosic Products/LIGNO, Itapeva, Brazil \\ ${ }^{4}$ University of São Paulo/EESC, Department of Materials Science and Engineering, São Carlos, Brazil \\ ${ }^{5}$ University of São Paulo/ESALQ, Department of Forest Sciences, Piracicaba, Brazil \\ ${ }^{6}$ University of São Paulo/EESC, Department of Structures Engineering, São Carlos, Brazil
}

\begin{abstract}
This study involved the study of physical and mechanical properties of Eucalyptus umbra wood for its use in timber structures. Two physical and fourteen mechanical properties tests were performed according to Brazilian code ABNT NBR 7190:1997. Two moisture contents were considered: above the Fiber Saturation Point (30\%) and standardized point $12 \%$. Decreasing the moisture content, this study presented an increase in eight mechanical properties. Oppositely, five mechanical properties presented decreasing of values, and one single variable persisted stable. But statistical perspective supported by the t-test, only three mechanical properties $\left(\mathrm{f}_{\mathrm{t} 90}, \mathrm{f}_{\mathrm{H} 0}\right.$ and $\left.\mathrm{W}\right)$ increased significantly. According to results obtained in this study, it is possible to use Eucalyptus umbra wood as raw material for structures.
\end{abstract}

Keywords Mechanical properties, Physical properties, Timber structures, Wood

\section{Introduction}

Wood is used as raw material in several sectors of economy, such as cellulose and paper production [1] and oriented strand board panels (OSB) [2]. For each purpose, their properties should be estimated, due to the variability found among different species [3] and, within the species itself, due to anatomical factors [4], edaphoclimatic [5] and moisture content [6].

For wood use as structural element in construction of bridges [7], trusses [8] among others [9], physical and mechanical properties are the most important [10-12].

In Brazil, the code ABNT NBR 7190:1997 [13] governs design of timber structural elements considering physical and mechanical properties determined by the procedures in its annex B.

Among species that can be used in civil construction, the most important are those belonging to genus Eucalyptus and Corymbia [14, 15]. Because of their rapid growth, eucalypt species have emerged among the major alternatives for lumber production in Brazil for the coming years [16].

Eucalyptus umbra is a species from Australia, with little diametric, Specific gravity, chemical constituents and

* Corresponding author:

alchristoforo@gmail.com (André L. Christoforo)

Published online at http://journal.sapub.org/ijme

Copyright (C) 2018 Scientific \& Academic Publishing. All Rights Reserved anatomical characteristics variabilities [17]. In addition, Eucalyptus umbra is resistant to attack by bio-deterioration agents [18].

This study aims to investigate the possibility of Eucalyptus umbra wood use in timber structures, through its physical and mechanical properties, estimated in two different moisture contents (12\% and $30 \%$ ) according to Brazilian code [13].

\section{Materials and Methods}

Eucalyptus umbra wood samples had origin in five logs collected in a planted forest at Rio Claro, Sao Paulo state, Brazil. These trees presented diameters from 0.17 to $0.26 \mathrm{~m}$ and 28 years old.

According to experimental procedures recommended by Brazilian code [13], the following physical and mechanical properties were estimated for Eucalyptus umbra wood:

- Specific gravity $\left(\rho_{\mathrm{b}}\right)$;

- Density $\left(\rho_{\text {ap }}\right)$;

- Compression parallel to grain strength $\left(\mathrm{f}_{\mathrm{c} 0}\right)$;

- Compression perpendicular to grain strength $\left(\mathrm{f}_{\mathrm{c} 90}\right)$;

- Tension parallel to grain strength $\left(\mathrm{f}_{\mathrm{t} t}\right)$;

- Tension perpendicular to grain strength $\left(\mathrm{f}_{\mathrm{t} 90}\right)$;

- Shear parallel to grain strength $\left(f_{v 0}\right)$;

- Cleavage parallel to grain strength $\left(\mathrm{f}_{\mathrm{s} 0}\right)$;

- Modulus of rupture in static bending $\left(f_{M}\right)$; 
- Modulus of elasticity in compression parallel to grain $\left(\mathrm{E}_{\mathrm{c} 0}\right)$;

- Modulus of elasticity in compression perpendicular to grain $\left(\mathrm{E}_{\mathrm{c} 90)}\right)$;

- Modulus of elasticity in tension parallel to grain $\left(E_{\mathrm{c} 0}\right)$;

- Modulus of elasticity in static bending $\left(E_{M}\right)$;

- Hardness parallel to grain $\left(\mathrm{f}_{\mathrm{H} 0}\right)$;

- Hardness perpendicular to grain $\left(\mathrm{f}_{\mathrm{H90}}\right)$;

- Toughness (W).

Two moisture content conditions were verified for wood samples: above saturation fiber point (30\% moisture content), and standardized point for structures [13] (12\% moisture content). For each property and density 6 specimens were made.

Considering that results for two moisture contents are independent and normal distributed, t-test was performed to verify differences among groups averages. Statistical hypotheses of t-test are established by two conditions, where averages do not differ $\left(\mathrm{H}_{0}: \mu_{1}=\mu_{2}\right)$ and the opposite $\left(\mathrm{H}_{1}: \mu_{1} \neq\right.$ $\left.\mu_{2}\right)$. In this study, data variances were considered different and unknown. Decision was focused on P-value and t-test significance level (5\%). $\mathrm{H}_{0}$ hypothesis was rejected when P-value was lower than 5\%. Statistical analyzes were carried out using $\mathrm{R}$ version 3.4 .2 software.

\section{Results and Discussions}

Table 1 shows, for all physical and mechanical properties of Eucalyptus umbra wood, and both moisture content (Mc), the average values $\left(\mathrm{x}_{\mathrm{m}}\right)$, standard deviations $(\mathrm{Sd})$ and t-test performed P-values.

Decreasing moisture content of Eucalyptus umbra wood, from $30 \%$ to $12 \%$, $\rho_{\text {ap }}$ decreased $0.13 \mathrm{~g} / \mathrm{cm}^{3}$, or $13 \%$ of its initial value. T-test analysis for $\rho_{\text {ap }}$ was rejected the null hypothesis of averages equivalence, i.e., density presented significant difference when moisture content decreased from $30 \%$ to $12 \%$ (P-value $<0.05$ ).

Along moisture content decrease condition, $\mathrm{f}_{\mathrm{c} 0}$ presented decrease of $6 \%$ (2.7 MPa) and $\mathrm{f}_{\mathrm{c} 90} 40 \%$ (2.1 MPa), as well as $\mathrm{f}_{\mathrm{t90}}$ presented increase of $60 \%$ (1.8 MPa) and $\mathrm{f}_{\mathrm{M}}$ increase of $7 \%(5.7 \mathrm{MPa}) . \mathrm{f}_{\mathrm{t} 0}$ did not present any variation. Through $\mathrm{t}$-test, only $\mathrm{f}_{\mathrm{t} 90}$ presented significant P-value, i.e., Tension parallel to grain strength presented significant difference when moisture content changed ( $\mathrm{P}$-value $<0,05)$.

For decreasing condition for moisture content, $\mathrm{E}_{\mathrm{c} 0}$ increased 6\% (853.1 MPa) and $\mathrm{E}_{\mathrm{M}}$ increased 3\% (394.3 $\mathrm{MPa}) . \mathrm{E}_{\mathrm{c} 90}$ and $\mathrm{E}_{\mathrm{t} 0}$ decreased around $41 \%$ (211.1 MPa) and 7\% (1277.3 MPa), respectively. T-test applied for modulus of elasticity indicated that all properties did not present significant P-value, i.e., modulus of elasticity did not present significant difference when moisture content changed (P-value $>0,05$ ).

$\mathrm{f}_{\mathrm{s} 0}$ decreased $20 \%(0.17 \mathrm{MPa}) . \mathrm{f}_{\mathrm{v} 0}, \mathrm{f}_{\mathrm{H} 90}, \mathrm{f}_{\mathrm{H} 0}$ and $\mathrm{W}$ increased 16\% (2.5 MPa), 64\% (1343 N), 28\% (324 N) and $16.89 \%(2.5 \mathrm{Nm})$, respectively. T-test for $\mathrm{f}_{\mathrm{s} 0}, \mathrm{f}_{\mathrm{vO}}, \mathrm{f}_{\mathrm{H} 90}, \mathrm{f}_{\mathrm{HO}}$ and
W resulted significant difference with moisture content decrease (P-value $<0,05)$.

Gonçalves et al. (2009) [19] studied mechanical properties of a hybrid Eucalyptus urophylla $x$ Eucalyptus grandis species, finding average values of $\mathrm{f}_{\mathrm{M}}, \mathrm{E}_{\mathrm{M}}, \mathrm{f}_{\mathrm{c} 0}$ and $\rho_{\mathrm{ap}}(12 \%$ moisture content) equal to 103.2 $\mathrm{MPa}, 12.474 \mathrm{MPa}, 67.38$ MPa and $0.56 \mathrm{~g} / \mathrm{cm}^{3}$, respectively. Eucalyptus umbra wood presented higher density and lower $\mathrm{f}_{\mathrm{c} 0}$ and $\mathrm{f}_{\mathrm{M}}$ values compared to hybrid Eucalyptus urophylla $x$ Eucalyptus grandis.

Table 1. Physical and mechanical properties of Eucalyptus umbra wood at $12 \%$ and $30 \%$ moisture contents

\begin{tabular}{|c|c|c|c|c|}
\hline Properties & Mc (\%) & $\mathbf{x}_{\mathrm{m}}$ & Sd & P-value \\
\hline \multirow{2}{*}{$\rho_{\mathrm{ap}}\left(\mathrm{g} / \mathrm{cm}^{3}\right)$} & 30 & 1.02 & 0.03 & \multirow{2}{*}{0.0042} \\
\hline & 12 & 0.89 & 0.08 & \\
\hline$\rho_{\mathrm{b}}\left(\mathrm{g} / \mathrm{cm}^{3}\right)$ & 12 & 0.70 & 0.05 & - \\
\hline \multirow{2}{*}{$\mathrm{f}_{\mathrm{c} 0}(\mathrm{MPa})$} & 30 & 45.4 & 9.0 & \multirow{2}{*}{0.4951} \\
\hline & 12 & 42.7 & 4.1 & \\
\hline \multirow{2}{*}{$\mathrm{f}_{\mathrm{c} 90}(\mathrm{MPa})$} & 30 & 5.2 & 1.8 & \multirow{2}{*}{0.0588} \\
\hline & 12 & 3.1 & 1.6 & \\
\hline \multirow{2}{*}{$\mathrm{f}_{\mathrm{t} 0}(\mathrm{MPa})$} & 30 & 90.4 & 26.9 & \multirow{2}{*}{1.0000} \\
\hline & 12 & 90.4 & 57.0 & \\
\hline \multirow{2}{*}{$\mathrm{f}_{\mathrm{t} 90}(\mathrm{MPa})$} & 30 & 1.2 & 1.4 & \multirow{2}{*}{0.0398} \\
\hline & 12 & 3.0 & 0.5 & \\
\hline \multirow{2}{*}{$\mathrm{f}_{\mathrm{M}}(\mathrm{MPa})$} & 30 & 78.4 & 15.1 & \multirow{2}{*}{0.6370} \\
\hline & 12 & 84.1 & 24.8 & \\
\hline \multirow{2}{*}{$\mathrm{E}_{\mathrm{c} 0}(\mathrm{MPa})$} & 30 & $13,723.7$ & 3620.5 & \multirow{2}{*}{0.6259} \\
\hline & 12 & $14,576.8$ & 2465.6 & \\
\hline \multirow{2}{*}{$\mathrm{E}_{\mathrm{c} 90}(\mathrm{MPa})$} & 30 & 519.3 & 179.7 & \multirow{2}{*}{0.0564} \\
\hline & 12 & 308.2 & 157.9 & \\
\hline \multirow{2}{*}{$\mathrm{E}_{\mathrm{t} 0}(\mathrm{MPa})$} & 30 & $17,089.7$ & 4086.5 & \multirow{2}{*}{0.6528} \\
\hline & 12 & $15,812.4$ & 5562.4 & \\
\hline \multirow{2}{*}{$\mathrm{E}_{\mathrm{M}}(\mathrm{MPa})$} & 30 & $13,916.5$ & 3499.8 & \multirow{2}{*}{0.8328} \\
\hline & 12 & $14,310.8$ & 3074.5 & \\
\hline \multirow{2}{*}{$\mathrm{f}_{\mathrm{v} 0}(\mathrm{MPa})$} & 30 & 13.10 & 2.40 & \multirow{2}{*}{0.1665} \\
\hline & 12 & 15.60 & 3.40 & \\
\hline \multirow{2}{*}{$\mathrm{f}_{\mathrm{s} 0}(\mathrm{MPa})$} & 30 & 0.86 & 0.14 & \multirow{2}{*}{0.0428} \\
\hline & 12 & 0.69 & 0.11 & \\
\hline \multirow{2}{*}{$\mathrm{f}_{\mathrm{H} 90}(\mathrm{~N})$} & 30 & 749 & 1.31 & \multirow{2}{*}{0.0672} \\
\hline & 12 & 2,092 & 1.41 & \\
\hline \multirow{2}{*}{$\mathrm{f}_{\mathrm{HO}}(\mathrm{N})$} & 30 & 851 & 0.91 & \multirow{2}{*}{0.0315} \\
\hline & 12 & 1,175 & 2.71 & \\
\hline \multirow{2}{*}{ W (Nm) } & 30 & 12.30 & 4.60 & \multirow{2}{*}{0.0012} \\
\hline & 12 & 14.80 & 5.40 & \\
\hline
\end{tabular}


Lima et al. (2011) [17] evaluated for 25 years-old Eucalyptus umbra trees from a forest area at Piracicaba city, in Sao Paulo State, $\rho_{\text {ap }} 0.621 \mathrm{~g} / \mathrm{cm}^{3}$ (12\% moisture content). Comparing literature values to the obtained in this study, their values were about $10 \%$ lower than the obtained here $\left(0.69 \mathrm{~g} / \mathrm{cm}^{3}\right)$.

Torres et al. (2016) [20] determined $\rho_{\mathrm{b}}, \rho_{\mathrm{ap}}, \mathrm{f}_{\mathrm{c} 0}$ and $\mathrm{E}_{\mathrm{c} 0}$ for Eucalyptus camaldulensis wood, resulting $0.47 \mathrm{~g} / \mathrm{cm}^{3}, 0.581$ $\mathrm{g} / \mathrm{cm}^{3}, 25.31 \mathrm{MPa}$ and 12,379 MPa, respectively. These results are lower than the ones determined for Eucalyptus umbra wood.

\section{Conclusions}

Through decrease of moisture content and emphasized condition of this study for Eucalyptus umbra wood, was verified the increasing of eight mechanical property values. In other hand five mechanical properties of this eucalypt species decreased their values. Furthermore, tension parallel to grain strength remained constant.

Statistically, among 14 mechanical properties of Eucalyptus umbra wood, only three properties $\left(\mathrm{f}_{\mathrm{t} 90}, \mathrm{f}_{\mathrm{H} 0}\right.$ and W) increased values significantly with moisture content change.

According to presented results for Eucalyptus umbra wood, it is possible to conclude the feasibility of its use as raw material for design of timber structures.

\section{ACKNOWLEDGMENTS}

Authors thank to Wood and Timber Structures Laboratory (LaMEM), Structural Engineering Department (SET), Sao Carlos Engineering School (EESC), Sao Paulo University, by the materials and resources used in this study.

\section{REFERENCES}

[1] Alves, I. C. N.; Gomide, J. L.; Colodette, J. L.; Silva, H. D. Caraterização tecnológica da madeira de Eucalyptus benthamii para parodução de celulose kraft. Ciência Florestal, 21(1), 167-174, 2011. DOI: 10.5902/198050982759.

[2] Gorski, L.; Cunha, A. B.; Rios, P. D.; Trianoski, R.; França, M. C.; Almeida, C. C. F.; Longo, B. L. Utilização da madeira de Eucalyptus benthamii na produção de painéis de partículas orientadas (OSB). Floresta, 45(4), 865-874, 2015.

DOI: $10.5380 /$ rf.v45i4.36280.

[3] Almeida, T. H.; Almeida, D. H.; Araújo, V. A.; Silva, S. A. M.; Christoforo, A. L.; Lahr, F. A. R. Density as estimator of dimensional stability quantities of Brazilian tropical woods. BioResources, 12(3), 6579-6590, 2017.

DOI: 10.15376/biores.12.3.6579-6590.

[4] Toong, W.; Ratnasingam, J.; Roslan, M. K. M.; Halis, R. The prediction of wood properties from anatomical characteristics: the case of common commercial Malaysian timbers. BioResources, 9(3), 5184-5197, 2014.
[5] Lahr, F. A. R.; Christoforo, A. L.; Silva, C. E. G.; Andrade Junior, J. R.; Pinheiro, R. V. Avaliação de propriedades físicas e mecânicas de madeiras de Jatobá (Hymenaea stilbocarpa Hayne) com diferentes teores de umidade e extraídas de regiões distintas Revista Árvore, 40(1), 147-154, 2016. DOI: 10.1590/0100-67622016000100016.

[6] Logsdon, N. B.; Calil Junior, C. Influência da umidade nas propriedades de resistência e rigidez da madeira. Cadernos de Engenharia de Estruturas, 18, 77-107, 2002.

[7] Scaliante, R. M.; Almeida, D. H.; Christoforo, A. L.; Calil Junior, C. Inspection to assessment of roundwood composite stringers bridge at Yolanda Farm in São Carlos city, Brazil. Revista Madeira: Arquitetura e Engenharia, 13(33), 27-36, 2012.

[8] Palludo, D. F.; Pinheiro, R. V.; Almeida, D. H.; Arroyo, F. N.; Almeida, T. H.; Takeda, M. C.; Christoforo, A. L.; Lahr, F. A. R. Timber Use in Truss structures for roof ("Howe" type -8 to 18 meters). International Journal of Materials Engineering, 7(5), 93-99, 2017. DOI: 10.5923/j.ijme.20170705.03.

[9] Calil Junior, C.; Molina, J. C. Coberturas em estruturas de madeira: exemplos de cálculo. São Paulo: Pini, 2010.

[10] Lahr, F. A. R.; Arroyo, F. N.; Almeida, T. H.; Almeida Filho, F. M.; Mendes, I. S.; Christoforo, A. L. Full characterization of Erisma uncinatum Warm wood specie. International Journal of Materials Engineering, 6(5), 147-150, 2017. DOI: 10.5923/j.ijme.20160605.01.

[11] Lahr, F. A. R.; Aftimus, B. H. C.; Arroyo, F. N.; Almeida, D. H.; Christoforo, A. L.; Chahud, E.; Branco, L. A. M. N. Full characterization of Vatairea sp wood specie. International Journal of Materials Engineering, 6(3), 92-96, 2016. DOI: 10.5923/j.ijme.20160603.05.

[12] Nascimento, M. F.; Almeida, D. H.; Almeida, T. H.; Christoforo, A. L.; Lahr, F. A. R. Physical and Mechanical Properties of Sabiá Wood (Mimosa caesalpiniaefolia Bentham.). Current Journal of Applied Science and Technology, 25(4), 1-5, 2017. DOI: 10.9734/CJAST/2017/3 8747.

[13] ABNT NBR 7190:1997. Projeto de estruturas de madeira. Rio de Janeiro, 1997.

[14] Lobão, M. S.; Lúcia, R. M. D.; Moreira, M. S. S.; Gomes, A. Caracterização das propriedades físico-mecânicas da madeira de Eucalipto com diferentes densidades. Revista Árvore, 28(6), 889-894, 2004. DOI: 10.1590/S0100-6762200400060 0014 .

[15] Pigozzo, J. C.; Arroyo, F. N.; Christoforo, A. L.; Calil Junior, C.; Lahr, F. A. R. Pull out strength evaluation of steel bars bonded-in to $45^{\circ}$ in round timbers of Corymbia citriodora treated with CCA. International Journal of Materials Engineering, 7(2), 25-32, 2017. DOI: 10.5923/j.ijme.201707 02.02 .

[16] Gonçalez, J. C.; Breda, L. C. S.; Barros, J. F. M.; Macedo, D. G.; Janin, G.; Costa, A. F.; Vale, A. T. Características tecnológicas das madeiras de Eucalyptus grandis W. Hill Ex Maiden e Eucalyptus cloeziana F. Muell visando ao seu aproveitamento na indústria moveleira. Ciência Florestal, 16(3), 329-341, 2006. DOI: 10.5902/198050981912.

[17] Lima, I. L.; Longui, E. L.; Garcia, R.; Luca, E. F.; Silva Júnior, F. G.; Florsheim, S. M. B. Propriedades da madeira de Eucalyptus umbra R. T. Baker em função do diâmetro e da 
posição radial na tora. Floresta e Ambiente, 18(3), 289-298, 2011. DOI: 10.4322/floram.2011.049.

[18] Zeni, T. L.; Silva, F. B.; Ferreira, M. M.; Magalhães, W. L. E.; Auer, C. G. Resistência natural das espécies Eucalyptus cloeziana, Eucalyptus mycrocoris, Eucalyptus umbra, Corymbia citriodora e Corymbia maculata à degradação provocada pelo fungo Agrocybe perfecta, causador da podridãobranca na madeira, In Vitro. In: Encontro Brasileiro em Madeiras e em Estruturas de Madeira (EBRAMEM), 10. Anais... São Pedro (SP), Brasil, 1-7, 2006.
[19] Gonçalves, F. G.; Oliveira, J. T. S.; Lucia, R. M. D.; Sartório, R. C. Estudo de algumas propriedades mecânicas da madeira de um híbrido clonal de Eucalyptus urophylla x Eucalyptus grandis. Revista Árvore, 33(3), 501-509, 20009. DOI: $10.1590 /$ S0100-67622009000300012.

[20] Torres, P. M. A.; Paes, J. B.; Nascimento, J. W. B.; Brito, F. M. S. Caracterização físico-mecânica da madeira jovem de Eucalyptus camaldulensis para aplicação na arquitetura rural. Floresta e Ambiente, 23(1), 109-117, 2016. DOI: $10.1590 / 2179-8087.016012$. 